\title{
Letter to the Editor concerning "Circumferential fusion using a custom-made screw in the management of high-grade spondylolisthesis" (by Jouve J-L et al. [2014] Eur Spine J; 23:S457-S462)
}

\author{
Marco Andrea Marino $^{1}$ - Davide Pasquetto ${ }^{1}$ Marco Scaglia $^{1} \cdot$ Pietro Bartolozzi $^{2}$ • \\ Bruno Magnan ${ }^{1}$
}

Received: 10 April 2015/Revised: 6 May 2015/Accepted: 6 May 2015/Published online: 13 May 2015

(c) Springer-Verlag Berlin Heidelberg 2015

Dear Sir,

We have read with great interest your article describing "an original" technique using a posterior-only approach to achieve a circumferential lumbosacral fusion with a custom-made screw [1]. Our attention was captured not only for the "innovative" surgical technique, but also for the fact that it came 11 years after our original article published in Spine [2]. Even the custom-made screw is very similar to our cage that we still use since 1997 . We know that in medicine it is very difficult to find new strategies, but is very important to learn from the past to find new treatment path and, in the meantime, to cite the correct references.
Conflict of interest None.

\section{References}

1. Jouve J-L, Blondel B, Fuentes S, Choufani E, Pesenti S, Bollini G (2014) Circumferential fusion using a custom-made screw in the management of high-grade spondylolisthesis. Eur Spine $\mathbf{J}$ 23:S457-S462

2. Bartolozzi P, Sandri A, Cassini M, Ricci M (2003) One-stage posterior decompression-stabilization and trans-sacral interbody fusion after partial reduction for severe L5-S1 spondylolisthesis. Spine 28:1135-1141
Marco Andrea Marino

m.marino76@gmail.com

Davide Pasquetto

davide.pasquetto@ospedaleuniverona.it

Marco Scaglia

drscagliam@gmail.com

Bruno Magnan

bruno.magnan@univr.it

1 Unità Operativa Complessa di Ortopedia e Traumatologia B, Ospedale Civile Maggiore, Azienda Ospedaliera Universitaria Integrata, Piazzale A. Stefani n.1, 37126 Verona, Italy

2 University of Verona, Verona, Italy 